\title{
Ultra-broadband near-infrared pulse generation by noncollinear OPA with angular dispersion compensation
}

\author{
T.-J. Wang • Z. Major • I. Ahmad • S.A. Trushin • \\ F. Krausz $\cdot$ S. Karsch
}

Received: 27 September 2009 / Published online: 1 November 2009

(C) The Author(s) 2009. This article is published with open access at Springerlink.com

\begin{abstract}
A scheme for the generation of an ultra-broadband, near-infrared beam from a Ti:sapphire source is proposed with the aim to serve as seed pulse of a petawatt-field synthesizer (Major et al. in Rev. Laser Eng. 37:431, 2009). The idler beam of a noncollinear optical parametric amplifier, pumped at the second harmonic of the Ti:sapphire output, displays the required bandwidth, albeit with an inherent angular chirp owing to the noncollinear geometry. We propose a scheme for the compensation of this angular dispersion which consists of a diffraction grating, a telescope and a deformable mirror. The suitability of this scheme is discussed quantitatively and preliminary experimental findings are shown.
\end{abstract}

PACS 42.65.Re $\cdot$ 42.65. Yj $\cdot$ 42.72.Ai $\cdot 42.79 . \mathrm{Nv}$

\section{Introduction}

The investigation of high-field laser-matter interactions requires the generation of laser pulses with which extreme

T.-J. Wang (凶) · Z. Major · I. Ahmad · S.A. Trushin · F. Krausz ·

S. Karsch

Max-Planck-Institut für Quantenoptik, Hans-Kopfermann-Str. 1,

85748 Garching, Germany

e-mail: tjwang2007@yahoo.com

Present address:

T.-J. Wang

Center for Optics, Photonics and Lasers (COPL), Laval

University, 2375 rue de la Terrasse, Quebec City, QC, G1V 0A6,

Canada

Z. Major · F. Krausz · S. Karsch

Department für Physik, Ludwig-Maximilians-Universität

München, Am Coulombwall 1, 85748 Garching, Germany peak intensities can be achieved, i.e. high pulse energies combined with ultra-short pulse durations, possibly in the few-cycle regime. In addition, for many experiments it is crucial that such high-power laser pulses have a very high temporal contrast, i.e. a ratio of $10^{8}-10^{10}$ between the main laser pulse and any prepulse or background, in order to avoid plasma generation by the pedestal of the pulse arriving before the main pulse. Conventional laser amplification combined with the chirped pulse amplification (CPA) scheme [2] allows for achieving laser pulses with peak-power levels up to the petawatt scale by amplifying a temporally stretched pulse and subsequent compression. However, the pulse duration of such pulses is limited by the laser material to a few tens of femtoseconds. In recent years the technique of optical parametric amplification (OPA), which does not suffer from this limitation and allows for very large amplification bandwidths, has opened up a new path towards generating ultra-high-power, few-cycle laser pulses [3-15]. High pulse energies can be obtained when combining the OPA technique with the CPA scheme (OPCPA), as first proposed by Dubietis et al. [16]. OPCPA systems have already been demonstrated to deliver pulse energies as high as $35 \mathrm{~J}$ in 84-fs pulses [17], 500-TW peak power for 30 -fs pulses [18] as well as $90 \mathrm{~mJ}$ in the few-cycle regime (10 fs) [19]. However, the generation of Joule-scale pulse energies in the fewcycle regime has yet to be demonstrated and constitutes the aim of the petawatt field synthesizer (PFS) [1] development.

At the heart of the PFS project lies the development of the novel short-pulse pumped OPCPA chain and its scaling to petawatt peak powers. The deuterated potassium dihydrogen phosphate (DKDP) crystals of the OPA stages will be pumped by the frequency-doubled light of a diode-pumped Yb:YAG pump laser, i.e. at $515 \mathrm{~nm}$. DKDP is available in sufficiently large apertures to allow for the high pulse energies required [18]. Since the broadest gain bandwidth of 
DKDP is centered around $900 \mathrm{~nm}$, an ultra-broadband seed pulse in the range of 700-1400 $\mathrm{nm}$ has to be used for the OPCPA chain. For optimum contrast of the output beam, the OPA stages should be seeded with a high-contrast pulse at an energy level as high as possible. As a common front end for the pump-laser chain and the main OPCPA chain a modified version of a commercially available Ti:sapphire source is used (for full details see [1, 20]). State-of-the art supercontinuum generation in a filament or hollow-core fiber can in principle generate a sufficiently broad spectrum, albeit in the range between $400 \mathrm{~nm}$ and $900 \mathrm{~nm}$, while a spectral shift towards the near infrared is hard to achieve when starting from an 800-nm pump source [21]. Moreover, the temporal contrast that can be achieved through these schemes has not been quantified so far and remains a reason for concern. The feasibility of generating the PFS seed pulse using plasma filaments, hollow-core fibers or a combination of both is detailed in [20].

In this study we propose to generate the broadband seed pulse in a noncollinear OPA stage using a beta barium borate (BBO) crystal, which is pumped by the second harmonic of the Ti:sapphire pulse and seeded by a spectrally broadened version of its fundamental. In our case the idler generated in the OPA can reach energies of several tens of $\mu \mathrm{J}$ and span the spectral range from $700 \mathrm{~nm}$ to $1400 \mathrm{~nm}$, which would be ideally suited for parametric amplification in DKDP. The idler also provides a temporal contrast which is inherently excellent. In principle there is no light emission outside the $\sim 50$-fs pump duration, given an ideal contrast of the pump. However, since the generation of the idler is nonlinear in the pump intensity, any precursors in the pump beam will only be transferred to the idler in a strongly suppressed way [22]. The main drawback of this approach is the fact that this near-infrared (NIR) idler pulse carries a large angular dispersion, requiring a complex compensation setup. A telescopegrating scheme, using a 600 lines/mm grating, has already been demonstrated in [23], but little attention was given to higher-order corrections needed in order to achieve best spatial and temporal focal quality of the final amplified pulses. We give a recipe for addressing these higher-order corrections by varying the parameters of the grating, telescope and deformable mirror, and present our preliminary experimental observations.

\section{Design considerations}

In optical parametric amplification a seed pulse is amplified by a three-wave mixing process in a nonlinear optical crystal, i.e. energy is transferred from the narrowband pump beam into the broadband seed beam. In this process the conservation of momentum and energy leads to the spontaneous generation of a third beam, the idler, as schematically indicated in Fig. 1. Since the amplification bandwidth only

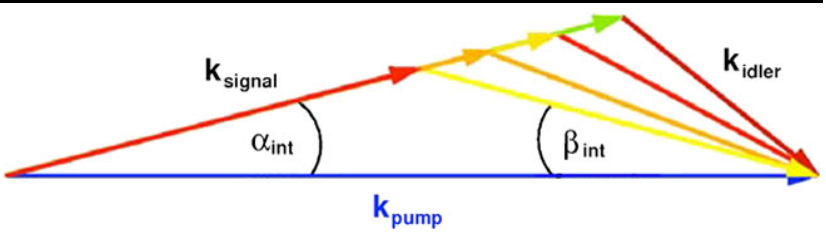

Fig. 1 Scheme of an OPA in noncollinear geometry (NOPA): pump, signal and idler fulfill energy and momentum conservation inside a nonlinear crystal resulting in an angular chirp of the emerging idler beam

depends on the phase matching between the seed and the pump beams in a fixed nonlinear crystal, amplification can occur over a very large bandwidth. If noncollinear geometry is chosen, this phase-matching bandwidth can be extended even further [3,5]; this is often referred to as noncollinear optical parametric amplification (NOPA).

In order to theoretically assess the feasibility of the idler beam generated in a NOPA as our ultra-broadband seed, and to estimate the achievable energy and bandwidth from the available PFS front end, we used a simple 1D model to calculate the three-wave mixing process of the OPA for our laser parameters [20,24]. The OPA is pumped with the second harmonic of the Ti:sapphire source, which is centered at $395 \mathrm{~nm}$, and the seed beam is assumed to be of Gaussian shape in frequency space resulting in a bandwidth ranging from 500 to $900 \mathrm{~nm}$. The available pump energy was assumed to be $\sim 300 \mu \mathrm{J}$; the pulse duration of the pump was taken to be $100 \mathrm{fs}$. Using a seed energy of $\sim 50 \mu \mathrm{J}$ in $\sim 80 \mathrm{fs}$, our OPA calculations in a $350-\mu \mathrm{m}$-thick BBO crystal yielded the following results. While the broadband amplified spectrum is shown in Fig. 2a, Fig. 2b shows the corresponding idler intensity spectrum, which clearly covers the spectral range we are aiming for. According to our calculations, the idler pulse energy can be up to $\sim 50 \mu \mathrm{J}$. The best amplification conditions could be obtained for a noncollinear angle (angle between pump and seed beam directions) of $3.5^{\circ}$ and a corresponding phase-matching angle of $31.2^{\circ}$ (angle between the pump direction and the optical axis of the crystal).

However, owing to the noncollinear geometry which is needed for this ultra-broadband amplification, the idler beam carries a significant angular chirp as schematically shown in Fig. 1. For a fixed input noncollinear angle inside the crystal $\alpha_{\text {int }}$, the generated internal idler angles $\beta_{\text {int }}$ are given by

$\beta_{\text {int }}=\arcsin \left(\frac{n_{s} \lambda_{i}}{n_{i} \lambda_{s}} \times \sin \alpha_{\text {int }}\right)$,

where $n_{s, i}$ and $\lambda_{s, i}$ are the refractive indices in the nonlinear crystal and the wavelengths of signal and idler beams, respectively. Taking into account refraction on the crystal 
Fig. 2 Calculated spectral intensity of the amplified signal (a) and the corresponding idler (b) resulting from NOPA in a BBO crystal, pumped at $395 \mathrm{~nm}$. The blue curves represent the input spectra, the red curves are the spectra after amplification

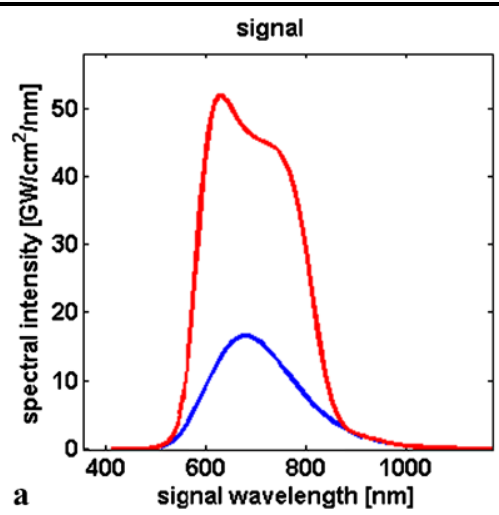

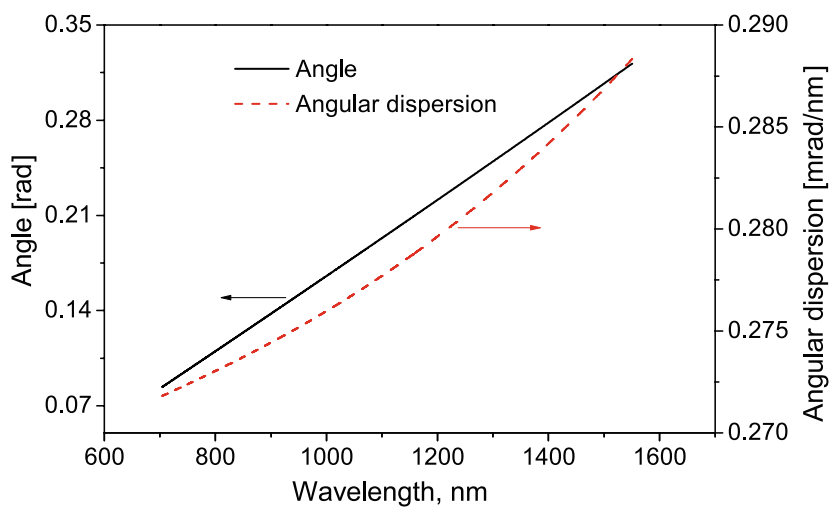

Fig. 3 Idler angle and angular dispersion from NOPA in a BBO crystal pumped at $395 \mathrm{~nm}$. For the spectral range of interest (700-1400 nm) an average angular chirp of $2.8 \mathrm{mrad} / \mathrm{nm}$ corresponds to a deviation in the propagation direction of up to $10^{\circ}(\sim 0.17 \mathrm{rad})$

surfaces and the conservation of energy, the external idler angle $\beta_{\text {ext }}$ is the following:

$\beta_{\text {ext }}=\arcsin \left(n_{i} \times \sin \beta_{\text {int }}\right)=\arcsin \left(B_{0} \times \lambda_{i}+B_{1}\right)$,

where $B_{0}=\frac{n_{s}}{\lambda_{p}} \sin \alpha_{\mathrm{int}}$ and $B_{1}=-n_{s} \sin \alpha_{\mathrm{int}}$.

Figure 3 shows the resulting external angular distribution as a function of wavelength of the idler beam for our experimental case where the pump wavelength is $395 \mathrm{~nm}$ and the noncollinear angle is close to $\alpha_{\mathrm{int}}=3.7^{\circ}$.

In order to further utilize the generated idler beam, this large angular chirp needs to be compensated, since, on one hand, with such a widespread distribution of exit angles it is impossible to propagate the idler for further amplification. On the other hand, when focused, even a small angular chirp can lead to a significant reduction of the intensity at the center of the focal spot. As elaborated in [25], this reduction has the following origins. Firstly, the angular chirp transforms into a spatial chirp in the far field, which results in a spatial broadening at the focal point. Secondly, the spectral bandwidth at each point in the focal plane is reduced by the spatial chirp, leading to a temporal broadening. A simple model reported in [25] can be used to estimate the effect of angular dispersion on the pulse duration and intensity in the focus. Here, the laser pulses are assumed to have a Gaussian intensity profile both spectrally and spatially and we also assume that the time-bandwidth product $(\Delta \lambda \Delta \tau)$ is unaffected by the angular chirp. The increase of the pulse duration can then be expressed as [25]

$\xi=\frac{\Delta \tau}{\Delta \tau_{0}}=\sqrt{1+\left(C_{a} \frac{\pi}{2 \ln 2} \frac{\Delta \lambda_{\mathrm{FWHM}}}{\lambda_{0}} d_{\mathrm{FWHM}}\right)^{2}}$,

where $\Delta \tau_{0}$ is the transform-limited pulse duration with a Gaussian spectrum with the width $\Delta \lambda_{\text {FWHM }}$ at the central wavelength $\lambda_{0} ; C_{a}$ is the angular chirp of the pulse and $d_{\text {FWHM }}$ is the width of the Gaussian intensity distribution in space. The elongated pulse duration and the enlarged focal spot produce a total intensity reduction in the focus center by a factor of $\xi^{2}$ [25].

The best possible compensation of the angular chirp is therefore crucial if the idler beam is to be used for driving any kind of high-field laser-matter interaction. Such angular chirp compensation of an idler beam produced in a NOPA scheme has already been demonstrated in [23]. Here the idler beam was imaged onto a diffraction grating which then compensated the angular chirp to a certain extent. Using a telescope the angular spread can be modified to match the angular dispersion introduced by the grating. Schematically, the principle of this compensation scheme is shown in Fig. 4. In the following we will evaluate the extent of angular chirp compensation of the scheme used in [23] and present a method for further improvement.

The angular distribution of a beam after passing a diffraction grating can be obtained from the grating equation

$n \times \lambda=d \times(\sin (\phi)+\sin (\theta))$,

where $\phi$ and $\theta$ are the incident and diffracted angles, respectively, $n$ is the diffraction order and $d$ is the grating constant. The angular distribution of a previously collimated beam after a grating is therefore given by

$\theta=\arcsin \left(\frac{n \times \lambda}{d}-\sin (\phi)\right)$. 


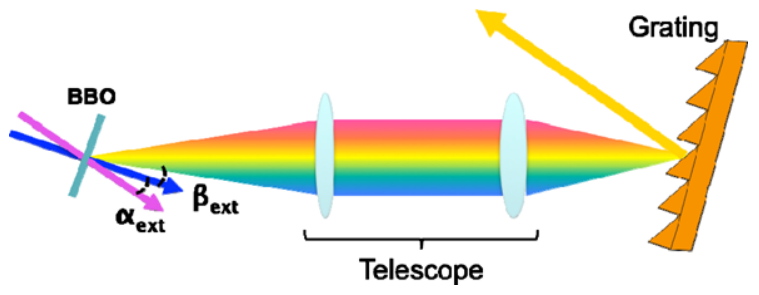

Fig. 4 Principle of an angular dispersion compensator based on a telescope and a grating. For clarity the imaging optics of the telescope are represented by lenses in the schematic picture

By equating the resulting angular distribution from the NOPA (2) with that from the diffraction grating (5) we can obtain the residual angle $R$ after the compensator:

$R\left(\lambda_{i}\right)=\beta_{\mathrm{ext}}\left(\lambda_{i}\right)-M \times \theta\left(\lambda_{i}\right)+$ constant.

The residual angular chirp after the compensator can be written as a Taylor series expanded around the central wavelength of the idler spectrum, $\lambda_{i 0}$. Considering terms up to second order in the Taylor series and diffraction by the grating into the first order $(n=1)$, these expressions read

$$
\begin{aligned}
R\left(\lambda_{i}\right) \approx & R\left(\lambda_{i 0}\right)+\left(\left.\frac{\partial \beta_{\mathrm{ext}}}{\partial \lambda_{i}}\right|_{\lambda_{i}=\lambda_{i 0}}-M \times\left.\frac{\partial \theta}{\partial \lambda_{i}}\right|_{\lambda_{i}=\lambda_{i 0}}\right) \\
& \times\left(\lambda_{i}-\lambda_{0}\right) \\
& +\left(\left.\frac{\partial^{2} \beta_{\mathrm{ext}}}{\partial \lambda_{i}^{2}}\right|_{\lambda_{i}=\lambda_{i 0}}-M \times\left.\frac{\partial^{2} \theta}{\partial \lambda_{i}^{2}}\right|_{\lambda_{i}=\lambda_{i 0}}\right) \\
& \times \frac{\left(\lambda_{i}-\lambda_{0}\right)^{2}}{2 !}+\cdots
\end{aligned}
$$

where

$$
\begin{aligned}
& \left.\frac{\partial \beta_{\mathrm{ext}}}{\partial \lambda_{i}}\right|_{\lambda_{i}=\lambda_{i 0}}=\left.\frac{B_{0}}{\sqrt{1-\left(B_{0} \lambda_{i}+B_{1}\right)^{2}}}\right|_{\lambda_{i}=\lambda_{i 0}}, \\
& \left.\frac{\partial^{2} \beta_{\mathrm{ext}}}{\partial \lambda_{i}^{2}}\right|_{\lambda_{i}=\lambda_{i 0}}=\left.\frac{B_{0}^{2}\left(B_{0} \lambda_{i}+B_{1}\right)}{\left(1-\left(B_{0} \lambda_{i}+B_{1}\right)^{2}\right)^{3 / 2}}\right|_{\lambda_{i}=\lambda_{i 0}}, \\
& \left.\frac{\mathrm{d} \theta}{\mathrm{d} \lambda_{i}}\right|_{\lambda_{i}=\lambda_{i 0}}=\left.\frac{1}{\sqrt{d^{2}-\left(\lambda_{i}-d \times \sin \phi\right)^{2}}}\right|_{\lambda_{i}=\lambda_{i 0}}, \\
& \left.\frac{\mathrm{d}^{2} \theta}{\mathrm{d} \lambda_{i}^{2}}\right|_{\lambda_{i}=\lambda_{i 0}}=\left.\frac{\lambda_{i}-d \times \sin \phi}{\left(d^{2}-\left(\lambda_{i}-d \times \sin \phi\right)^{2}\right)^{\frac{3}{2}}}\right|_{\lambda_{i}=\lambda_{i 0}} .
\end{aligned}
$$

$M$ denotes the magnification factor of the telescope. In the above calculation of the expansion coefficients, we have assumed the dependence of $n_{s}$ on $\lambda_{i}$ to be constant, which allows us to neglect the partial derivatives of $n_{s}$. Some further algebra and the comparison with $B_{0}$ justify this assumption. With the external idler angle $\beta_{\text {ext }}$ (2), we can see from (7) that for a given grating (grating constant $d$ and the corresponding incident angle $\phi$ ), the linear term of the angular chirp vanishes if the magnification of the telescope is chosen appropriately. If we allow for an arbitrary grating constant, $M$ and $d$ can be chosen at a given $\phi$ so that both the linear and the quadratic terms vanish in the above expressions. However, this analysis does not account for any higher-order terms, since there are only two free parameters ( $M$ and $d$ ), allowing for the control of at most two orders. This is also the reason why our series expansion is truncated at the second order. The residual angular spread resulting from these first two terms amounts to a few mrad, which can be obtained by inserting the parameters from our experimental design into (6). For applications that target a small overall bandwidth this purely analytical approach might be sufficient, but for our ultra-broadband case the residual errors are too large. Hence, we applied an alternative approach to find the optimum values for the magnification factor of the telescope and the grating constant, for which also the higherorder terms are reduced. Using a least-squares fit we minimized (6) and thereby obtained optimum values for $M$ and $d$, for which all orders have a combined minimum contribution to the residual angular spread. Note that the shapes of the resulting residual angular dispersion curves sensitively depend on the wavelength range used for the fitting. In our calculations, we chose the wavelength range to be 700 $1550 \mathrm{~nm}$, which corresponds to the signal wavelength range of $530-900 \mathrm{~nm}$.

For our idler with a central wavelength of $1100 \mathrm{~nm}$ and a 600-nm spectral bandwidth, the fitting results in an optimum groove density of 221 lines $/ \mathrm{mm}$ for the grating and a magnification factor of 1.22 for the telescope. The corresponding angle of incidence is $\phi=-2^{\circ}$. The residual angle difference is at most $\sim 3 \mu \mathrm{rad}$ and the corresponding angular dispersion is less than $0.04 \mu \mathrm{rad} / \mathrm{nm}$ as shown in the left-hand column of Fig. 5. This optimized residual angular dispersion is two orders of magnitude smaller than that reported in [23]. However, such specialized gratings may be hard to come by. We have therefore investigated the case of a commercial grating with 300 lines $/ \mathrm{mm}$. For this fixed grating constant the optimum magnification of the telescope is 0.91 , the angle of incidence is $\phi=6.7^{\circ}$ and the calculated residual angular spread and angular dispersion curves are depicted in the right-hand column of Fig. 5. From these we can see that the residual angular dispersion is $\sim 50 \mu \mathrm{rad}$, and thus still one order or magnitude below that in [23]. Comparison with our Taylor series expansion analysis shows, that the higherorder terms play a significant role, since setting the linear and quadratic terms to zero still results in a residual angular spread one to two orders of magnitude larger than that obtained from the fit.

Using expression (3) we can now estimate the effect of the residual angular chirp for the different cases we have investigated. In Fig. 6 the resulting pulse duration is shown as a function of angular chirp in the beam. We have assumed 

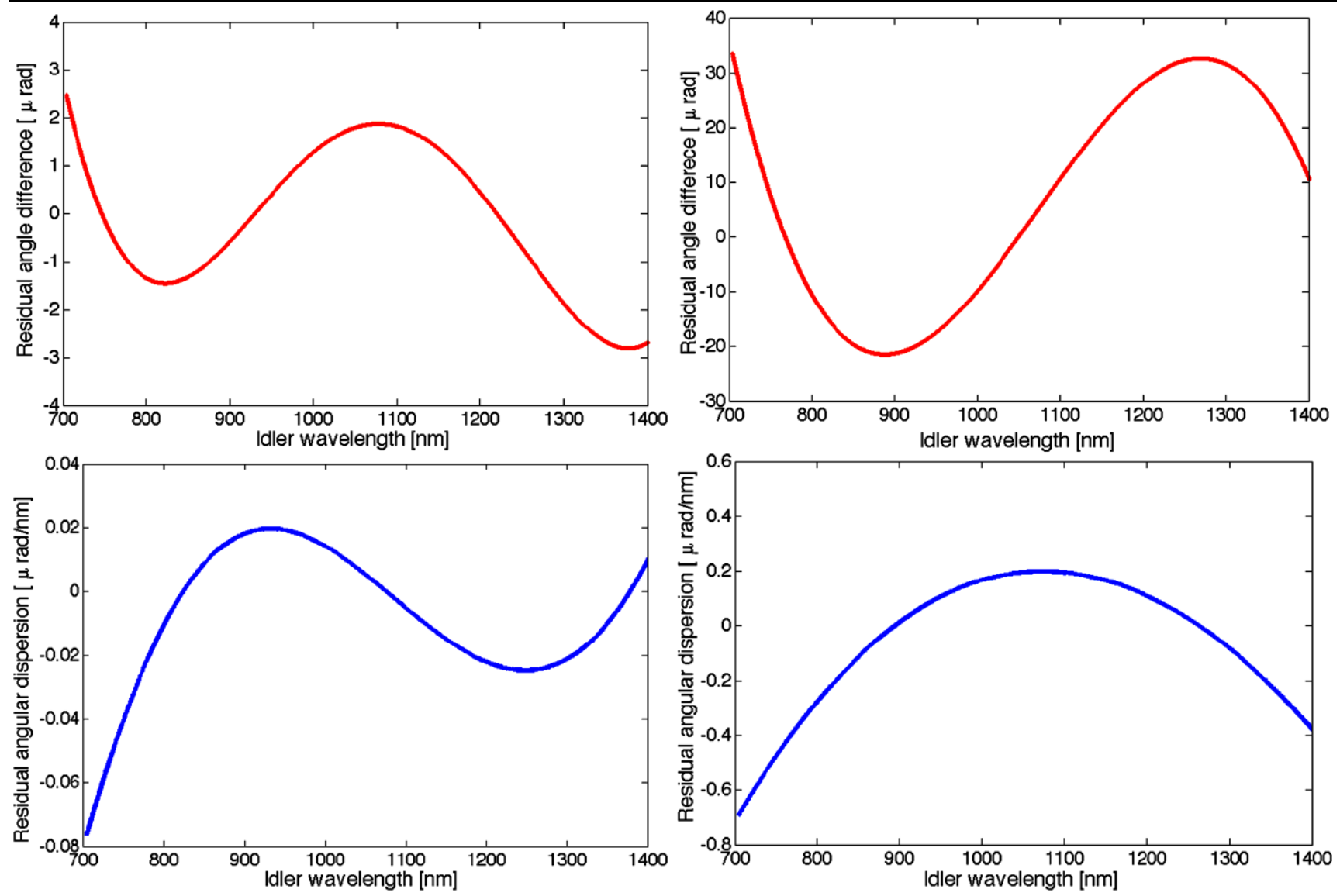

Fig. 5 Residual angular spread (top) and angular dispersion (bottom) resulting from the least-squares fitting. The left-hand column shows the results of the best fit when the grating constant is also a fit parameter,

the right-hand column shows the results when the grating constant is kept fixed at 300 lines $/ \mathrm{mm}$

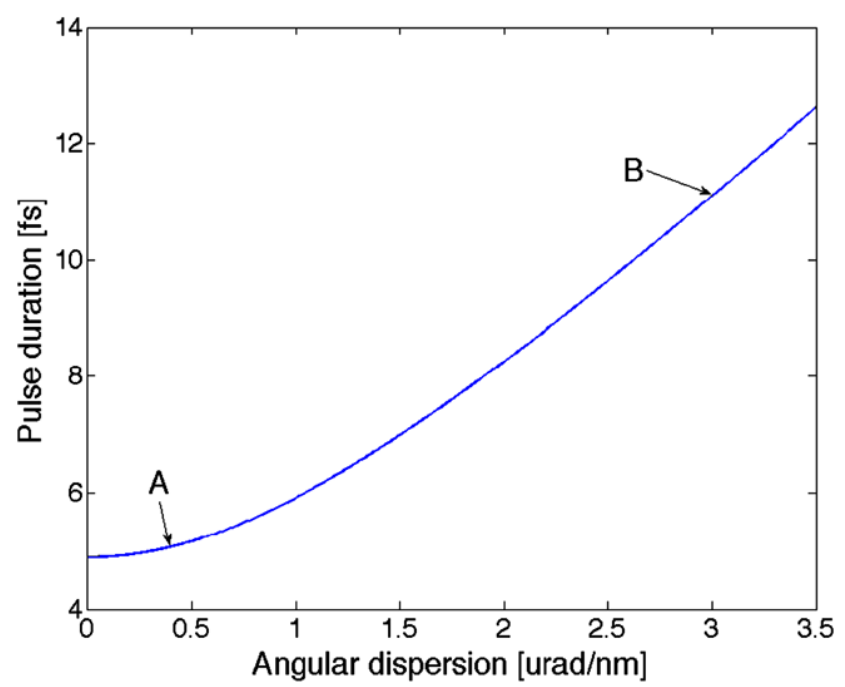

Fig. 6 Effect of angular dispersion on the pulse duration in the focus according to (3). ' $A$ ' indicates the case of $0.4 \mu \mathrm{rad} / \mathrm{nm}$ residual angular chirp with the 300 lines/mm grating, ' $B$ ' corresponds to the case reported in [23] using a grating with 600 lines $/ \mathrm{mm}$. A transform-limited pulse with 300-nm spectral bandwidth, 1-mm beam size in FWHM, at the central wavelength of $1000 \mathrm{~nm}$ is considered in the calculation a pulse with a Gaussian spectrum with 300-nm bandwidth, i.e. 4.9-fs transform-limited pulse duration, a beam size of $1 \mathrm{~mm}$ in FWHM and a central wavelength around $1000 \mathrm{~nm}$. With $0.4 \mu \mathrm{rad} / \mathrm{nm}$ angular dispersion (corresponding to the 300 lines/mm grating) the pulse duration increases by $4 \%$ to $\sim 5.1$ fs as marked by 'A'. However, the same pulse is stretched to $\sim 11$-fs duration if the angular chirp is one order of magnitude higher as in the case reported in [23], indicated by ' $\mathrm{B}$ '. We have also verified the validity of our fitted results using the 221 and 300 lines/mm gratings for a realistic experimental situation modeled by ray-tracing analysis using the code RAYTRACE [26].

This rather simple angular dispersion compensation scheme based on a telescope and a diffraction grating cannot exactly compensate the entire angular chirp of the idler beam. However, for cases when $0.4 \mu \mathrm{rad} / \mathrm{nm}$ residual angular dispersion, corresponding to a few tens of $\mu \mathrm{rad}$ in angular spread, is still too large for the respective applications, this remainder can be compensated using a deformable mirror placed in the Fourier plane of the telescope. The necessary deformation could be achieved in practice with a commercial mirror (e.g. [27]), which can introduce a maximum cor- 
rection angle around $\pm 320 \mu \mathrm{rad}$ with a maximum stroke of $8 \mu \mathrm{m}$, resulting in a maximum correctable gradient of $0.2 \mu \mathrm{m} / \mathrm{mm}$. Thereby, this simple scheme could easily be extended such that even higher orders of the angular dispersion can be fully compensated.

\section{Experimental setup}

Figure 7 shows the schematic experimental setup for the idler generation in the NOPA followed by the angular chirp compensation. Since the final goal of our experiment is to produce an ultra-broadband, high-energy, high-contrast seed pulse for the short-pulse-pumped OPCPA chain of PFS, we used its already available front end, a modified Femtopower Compact Pro system (Femtolasers $\mathrm{GmbH}$ ). This consists of a Ti:sapphire oscillator and multipass amplifier with a sub-

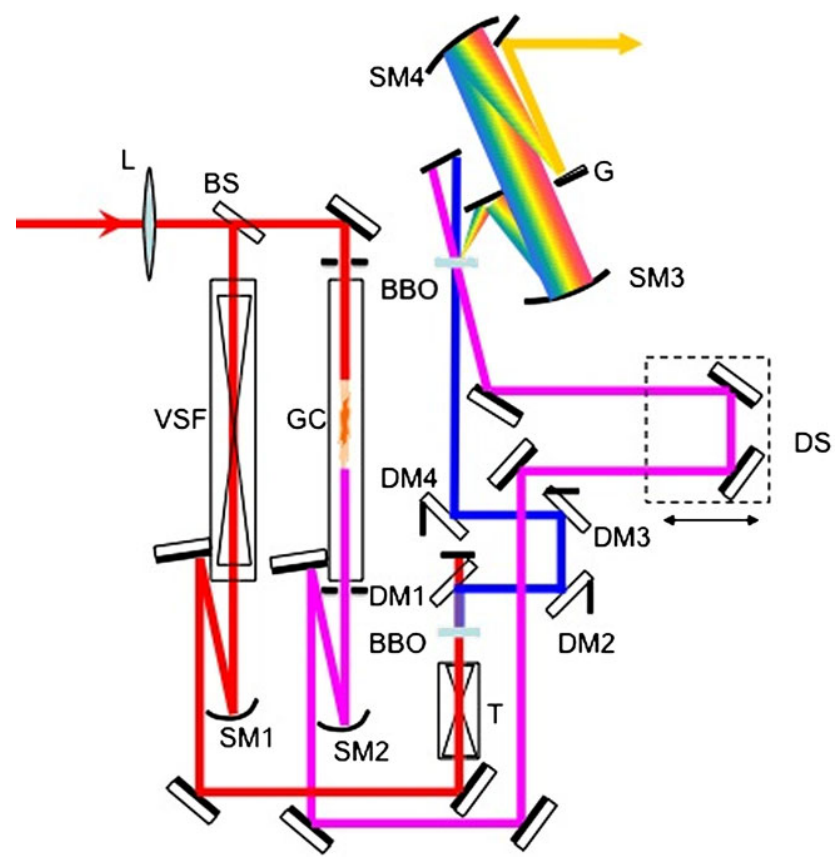

Fig. 7 Schematic experimental setup (see text for details). L: lens; $B S$ : beam splitter; $V S F$ : vacuum spatial filter; $S M 1-4$ : spherical mirrors; $T$ : telescope; $D M 1-4$ : dielectric mirrors; $G C$ : gas cell; $D S$ : delay line; $G$ : diffraction grating sequent hybrid pulse compressor [20], delivering up to 1.4$\mathrm{mJ}$ pulses with a duration of around $30 \mathrm{fs}$ centered around $800 \mathrm{~nm}$ at a repetition rate of $1 \mathrm{kHz}$.

After passing through a focusing lens $\mathrm{L}(f=1.5 \mathrm{~m})$ the beam was divided into two parts by a beam splitter BS $(65 / 35)$. The reflected part propagated through a vacuum spatial filter VSF and was collimated by a spherical silver mirror SM1 $(f=1 \mathrm{~m})$ after its exit. A reflectivetype telescope $\mathrm{T}$ was used to change the beam size. After a thin BBO crystal $(120 \mu \mathrm{m})$ cut for Type I frequency doubling at $800 \mathrm{~nm}\left(\theta=29.2^{\circ}\right)$, four dielectric mirrors DM1-4 coated for maximum reflectivity at $400 \mathrm{~nm}$ were used to separate the fundamental beam from the second harmonic, which was then used to pump the NOPA stage. The fraction of the pulse, which was transmitted through the beam splitter, was spectrally broadened in a filament in a gas cell GC filled with argon at a pressure of 2 bar. An iris was used to select the central part of the beam after having formed a single filament. After collimation by a spherical silver mirror SM2 $(f=1.25 \mathrm{~m})$ and a delay line DS, the white light was used to seed the NOPA. We used a $500-\mu \mathrm{m}$ thick Type I BBO crystal $\left(\theta=31^{\circ}\right)$ and an internal noncollinear angle of around $3.7^{\circ}$ between the pump and seed directions. In addition to the amplified broadband pulse, an angularly dispersed idler was generated. The idler was first deflected by a silver-coated rectangular mirror guiding it into an imaging telescope which consisted of two spherical silver mirrors SM3, 4 (ROC $=400 \mathrm{~mm}, 365 \mathrm{~mm})$. The telescope imaged the NOPA crystal onto the diffraction grating $\mathrm{G}$ (300 lines/mm), which was used to compensate the angular dispersion. After the compensator the collimated idler was picked out by another flat silver mirror.

In our preliminary experiment the total pulse energy of the idler after passing through the compensator setup was $3 \mu \mathrm{J}$, with a pump energy of $\sim 160 \mu \mathrm{J}$ and an input seed energy of $40 \mu \mathrm{J}$. The seed pulse was amplified to $60 \mu \mathrm{J}$. The typical spectra for the input seed signal, amplified signal and compensated idler are shown in Fig. 8, measured with a combined visible-NIR spectrometer (AvaSpec-NIR2561.7). Note that the 'kink' in the idler spectrum originates from the overlapping region between the ranges of the two spectrometers. From Fig. $8 \mathrm{~b}$ we can see that the generated
Fig. 8 (a) Spectrum of the seed pulse before (black) and after (red) amplification in the NOPA; (b) spectrum of the idler after the angular dispersion compensator
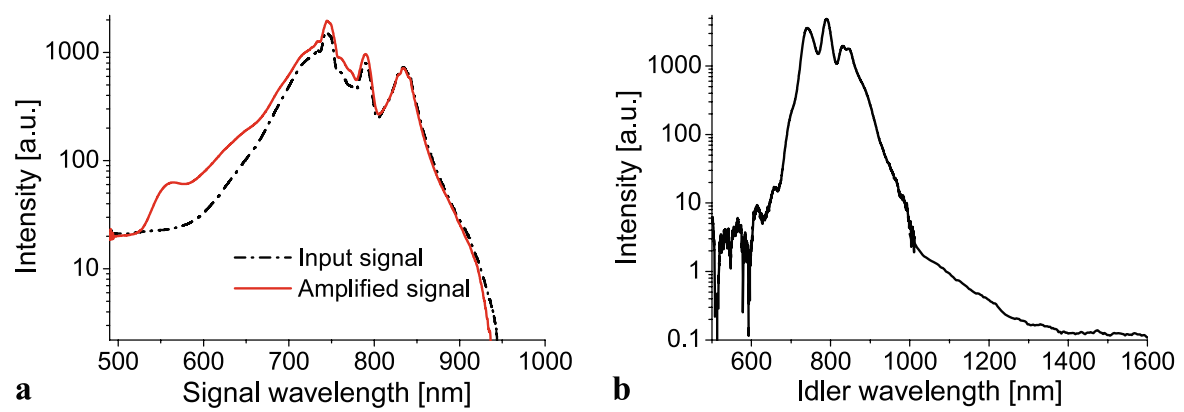
idler ranges from below $700 \mathrm{~nm}$ up to around 1400-nm wavelength. However, the spectral intensity in the NIR tail of the idler spectrum is up to four orders of magnitude lower than its peak around $800 \mathrm{~nm}$, originating firstly from the amplification process itself and secondly from the diffraction efficiency of the grating. In an improved experimental setup the first issue could be overcome by increasing the spectral intensity of the seed at lower wavelengths, where the filament only provides an exponential tail, by using a hollow-core fiber instead of a filament for spectral broadening. Alternatively, or in addition, the amplification in the NOPA stage can be tuned such that the low-intensity, lowwavelength part of the seed experiences a very high gain, while the rest of the spectrum is already in saturation as shown in a similar setup in [24]. This would result in an increase in the long-wavelength part of the idler and thereby lower the intensity variations across its entire spectral range.

In order to assess the capability of our optimized angular dispersion compensation setup the beam profiles were recorded with a CCD camera (WinCamD) device after passing through the compensator and are shown in Fig. 9. For comparison, Fig. 9a shows the focal spot of the output of the PFS front end measured at the VSF. Figure 9b shows the focus of the idler beam with $\sim 2$-mm diameter after the compensator when focused by a lens with $f=12.5 \mathrm{~cm}$. After the compensator, when the residual angular chirp seems to be minimized, the beam is divergent. The profiles of the near field and the far field (after $1 \mathrm{~m}$ of propagation) of this beam are shown in Fig. 9c and d, respectively. Although the silicon-based chip of the CCD camera which was used is not sensitive to spectral components above $\sim 1000 \mathrm{~nm}$, and in spite of the low intensity of the NIR tail of the idler spectrum, we can conclude from the observed beam profiles
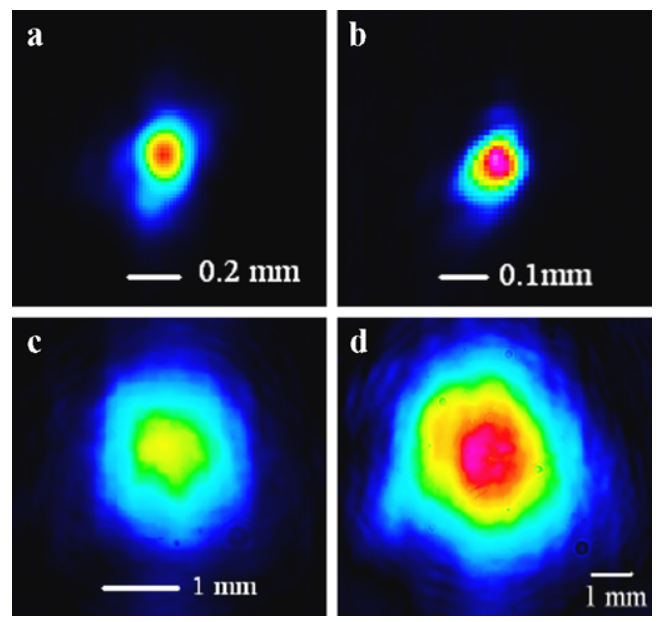

Fig. 9 Beam profiles of seed and compensated idler: (a) the focused beam pattern from the modified Femtopower Pro; (b) the compensated idler at the focus; (c) and (d) the patterns at near field and far field at 1-m distance without focusing. All the profiles are shown on the same spatial scale that the majority of the angular chirp of the idler has been compensated. For an uncompensated angular dispersion we would observe a significant broadening along one dimension of the beam profile after some propagation, even for the limited bandwidth that we are able to detect with this method. However, with this equipment it is not possible to quantitatively determine and therefore minimize the residual angular chirp after compensation. For this, additional measurements would be necessary, such as quantitatively measuring the angular dispersion of the idler using a technique based on spectrally resolved interferometry [28].

\section{Summary}

We have proposed an improved scheme for compensating the inherent angular chirp of an idler pulse generated in a NOPA stage based on the combination of a telescope and a diffraction grating. By optimizing the values of the magnification factor of the telescope and by choosing the appropriate grating constant, our analysis showed that the residual angular dispersion of the idler can be reduced to a value at least one order of magnitude below that achieved to date [23]. If few-cycle pulses are considered it is crucial to minimize the residual angular chirp, since it can lead to a significant temporal broadening and therefore a reduced intensity in the focus of the pulse. For the remaining higherorder terms of the angular dispersion we propose to apply a deformable mirror within the compensator setup.

We have experimentally demonstrated that the NOPA pumped at the second harmonic of a Ti:sapphire source and seeded by the spectrally broadened output of a filament is capable of generating an idler beam in the desired wavelength range of 700-1400 $\mathrm{nm}$. However, the setup and the amplification process can still be optimized for a more uniform spectral intensity distribution across this large spectral range. In addition to our preliminary studies confirming the reduction of the angular chirp of the idler by the proposed compensator design, more quantitative investigations are needed for the full verification, which are currently under way.

Open Access This article is distributed under the terms of the Creative Commons Attribution Noncommercial License which permits any noncommercial use, distribution, and reproduction in any medium, provided the original author(s) and source are credited.

\section{References}

1. Zs. Major, S.A. Trushin, I. Ahmad, M. Siebold, C. Wandt, S. Klingebiel, T.-J. Wang, J.A. Fülöp, A. Henig, S. Kruber, R. Weingartner, A. Popp, J. Osterhoff, R. Hörlein, J. Hein, V. Pervak, A. Apolonski, F. Krausz, S. Karsch, Rev. Laser Eng. 37, 431 (2009) 
2. D. Strickland, G. Mourou, Opt. Commun. 56, 219 (1985)

3. G.M. Gale, M. Cavallari, T.J. Driscoll, F. Hache, Opt. Lett. 20, $1562(1995)$

4. T. Wilhelm, J. Piel, E. Riedle, Opt. Lett. 22, 1494 (1997)

5. I.N. Ross, P. Matousek, M. Towrie, A.J. Langley, J.L. Collier, Opt. Commun. 144, 125 (1997)

6. G. Cerullo, M. Nisoli, S. Stagira, S. De Silvestri, Opt. Lett. 23, 12835 (1998)

7. A. Shirakawa, I. Sakane, M. Takasaka, T. Kobayashi, Appl. Phys. Lett. 74, 2268 (1999)

8. M.R. Armstrong, P. Plachta, E.A. Ponomarev, R.J.D. Miller, Opt. Lett. 26, 1152 (2001)

9. A. Baltuska, T. Kobayashi, in Parametric Amplification and Phase Control of Few-Cycle Light Pulses Few-Cycle Laser Pulse Generation and Its Applications, ed. by F.X. Kärtner (Springer, Berlin, 2004), pp. 179-228

10. N. Ishii, L. Turi, V.S. Yakovlev, T. Fuji, F. Krausz, A. Baltuska, R. Butkus, G. Veitas, V. Smilgevicius, R. Danielius, A. Piskarskas, Opt. Lett. 30, 567 (2005)

11. S. Witte, R.Th. Zinkstok, W. Hogervorst, K.S.E. Eikema, Opt. Express 13, 4903 (2005)

12. S. Witte, R.Th. Zinkstok, A.L. Wolf, W. Hogervorst, W. Ubachs, K.S.E. Eikema, Opt. Express 14, 8168 (2006)

13. C. Vozzi, F. Calegri, E. Benedetti, S. Gasiov, G. Sansone, G. Cerullo, M. Nisoli, S. De Silvestri, S. Stagira, Opt. Lett. 32, 2957 (2007)

14. S. Adachi, H. Ishii, T. Kanai, N. Ishii, A. Kosuge, S. Watanabe, Opt. Lett. 32, 2487 (2007)

15. D. Brida, G. Cirmi, C. Manzoni, S. Bonora, P. Villoresi, S. De Silvestri, G. Cerullo, Opt. Lett. 33, 741 (2008)

16. A. Dubietis, G. Jonusauskas, A. Piskarskas, Opt. Commun. 88, 437 (1992)
17. O.V. Chekhlov, J.L. Collier, I.N. Ross, P.K. Bates, M. Notley, C. Hernandez-Gomez, W. Shaikh, C.N. Danson, D. Neely, P. Matousek, S. Hancock, L. Cardoso, Opt. Lett. 31, 3665 (2006)

18. V.V. Lozhkarev, G.I. Freidman, V.N. Ginzburg, E.V. Katin, E.A. Khazanov, A.V. Kirsanov, G.A. Luchinin, A.N. Mal'shakov, M.A. Martyanov, O.V. Palashov, A.K. Poteomkin, A.M. Sergeev, A.A. Shaykin, I.V. Yakovlev, Laser Phys. Lett. 4, 421 (2007)

19. F. Tavella, A. Marcinkevicius, F. Krausz, Opt. Express 14, 12822 (2006)

20. I. Ahmad, S.A. Trushin, Zs. Major, C. Wandt, S. Klingebiel, T.J. Wang, V. Pervak, A. Popp, M. Siebold, F. Krausz, S. Karsch, Appl. Phys. B (2009). doi:10.1007/s00340-009-3599-4

21. E. Goulielmakis, S. Koehler, B. Reiter, M. Schultze, A.J. Verhoef, E.E. Serebryannikov, A.M. Zheltikov, F. Krausz, Opt. Lett. 33, 1407 (2008)

22. R.C. Shah, R.P. Johnson, T. Shimada, K.A. Flippo, J.C. Fernandez, B.M. Hegelich, Opt. Lett. 34, 2273 (2009)

23. A. Shirakawa, I. Sakane, T. Kobayashi, Opt. Lett. 23, 1292 (1998)

24. J.A. Fülöp, Zs. Major, A. Henig, S. Kruber, R. Weingartner, T. Clausnitzer, E.-B. Kley, A. Tünnermann, V. Pervak, A. Apolonski, J. Osterhoff, R. Hörlein, F. Krausz, S. Karsch, New J. Phys. 9, 438 (2007)

25. G. Pretzler, A. Kasper, K.J. Witte, Appl. Phys. B 70, 1 (2000)

26. Optical design program RAYTRACE, ver. 0.9(12), University of Erlangen-Nuremberg, http://www.optik.uni-erlangen.de/odem/

27. http://www.okotech.com/linear

28. K. Varjú, A.P. Kovács, G. Kurdi, K. Osvay, Appl. Phys. B 74(Suppl.), S259 (2002) 